\title{
Partial molecular volumes of cholesterol and phosphatidylcholine in mixed bilayers
}

Abstract Dispersion of multilamellar liposomes of dimyristoylphosphatidylcholine (DMPC) and cholesterol (CHOL) were studied by vibrational densitometer for the $\mathrm{CHOL}$ mole fractions $X=0-0.54$ in the temperature range $18-50{ }^{\circ} \mathrm{C}$, both below and above the main phase transition. DMPC-CHOL bilayers served as a simple model for lipidic part of biological membrane. Volumetric parameters are essential not only to evaluate the data obtained by scattering and diffraction methods on model membranes but can provide valuable information about molecular packing in bilayers and the phase behaviour of lipid-CHOL mixtures. In this paper, preliminary results regarding the changes in the specific volume of lipid bilayer with increasing temperature and $\mathrm{CHOL}$ content are presented. Different values of apparent molecular volume of CHOL for different CHOL mole fraction pointed out the non-ideal mixing of DMPC and CHOL.

Keywords -cholesterol, dimyristoylphosphatidylcholine, specific volume, densitometry

\section{INTRODUCTION}

In biological systems, $\mathrm{CHOL}$ is known to play a fundamental role as a modulator of physical properties and lateral organization of the membrane lipid bilayer. Thus, many studies of the interaction of $\mathrm{CHOL}$ with phospholipid bilayer model membranes have been performed, utilizing a wide range of physical techniques (for a review, see Marquardt et al., 2016). In our recent paper (Gallová et al., 2015), we determined the molecular volumes of $\mathrm{CHOL}$ and of a series of monounsaturated diacylphosphatidylcholines with the number of carbon atoms in the acyl chain 16, 18, 20, 22 and 24 in the temperature range $20-40^{\circ} \mathrm{C}$ in mixed bilayer. This work is devoted to the preliminary study of volumetric parameters of DMPC and CHOL in mixed bilayers. The knowledge of these parameters is essential to evaluate the data obtained by scattering and diffraction methods on model membranes. Greenwood et al. (2006) studied the binary systems of CHOL with different phospholipids at limited temperature points. In this paper, changes in volumetric parameters of $\mathrm{CHOL}$ and DMPC in mixed bilayers induced by increasing temperature and $\mathrm{CHOL}$ content are studied.

\section{MATERIAL AND METHODS}

Chemicals: 1,2-dimyristoyl-sn-glycero-3-phosphocholine (DMPC) was purchased from Avanti Polar Lipids (Alabaster, Alabama), CHOL from Sigma Aldrich (Germany) and chloroform, p.a. from Slavus (Bratislava, Slovakia).

Sample preparation: DMPC and CHOL were weighed in a dry state into a glass vial and then co-dissolved with a small amount of chloroform. The solution was mixed thoroughly. The solvent was evaporated to dryness under a stream of gaseous nitrogen, followed by evacuation in a vacuum chamber. The dry lipid film was hydrated by the weighed amounts of deionised water to obtain the final concentration of lipid $\approx 1-3 \%(w / w)$. Multilamellar liposomes were formed during vortexing and brief sonication in a bath sonicator. Samples were heated to $30{ }^{\circ} \mathrm{C}$ and degassed before measurement. The density of prepared samples and also of deionised water was measured using vibrational densitometer DMA 4500M (Anton Paar, Austria) in the temperature range $16-50^{\circ} \mathrm{C}$ with step $1{ }^{\circ} \mathrm{C}$. Weighing errors were $\pm 0.0001 \mathrm{~g}$; the precision of the density measurement was $\pm 0.00005 \mathrm{~g} / \mathrm{cm}^{3}$.

\footnotetext{
${ }^{*}$ E-mail: gallova@fpharm.uniba.sk

(c) European Pharmaceutical Journal
} 
Data treatment: The specific volume of the sample, $v_{s^{\prime}}$ is a reciprocal value of the sample density measured by a densitometer. According to (Greenwood et al., 2006; Klacsová et al., 2010) we assumed that the specific volume of water in our samples is the same as in pure bulk water. Under this precondition, the apparent specific volume of DMPC, $v_{P C^{\prime}}$ was calculated from the specific volume of the control sample $v_{s 0}$ without cholesterol, according to the equation 1

$v_{P C}=\frac{v_{S 0}-\left(1-w_{P C}\right) v_{W}}{w_{P C}}$

where, $v_{w}$ is the specific volume of water and $w_{P C}$ is the mass fraction of DMPC in the control sample defined as

$w_{P C}=\frac{m_{P C}}{m_{P C}+m_{W}}$

where, $m_{P C}$ and $m_{w}$ are masses of DMPC and water in the sample, respectively. The apparent specific volume of the nonaqueous part (DMPC + cholesterol) of the sample, $v_{P C+C H^{\prime}}$ was calculated in a similar way:

$v_{P C+C H}=\frac{v_{S}-\left(1-w_{P C+C H}\right) v_{W}}{w_{P C+C H}}$

where, $w_{P C+C H}$ is the mass fraction of lipid part in the sample defined as

$w_{P C+C H}=\frac{m_{P C}+m_{C H}}{m_{P C}+m_{C H}+m_{W}}$

If we further suppose that the specific volume of DMPC in the sample does not depend on the $\mathrm{CHOL}$ content, the apparent specific volume of $\mathrm{CHOL}, \mathrm{v}_{\mathrm{CH}^{\prime}}$ can be calculated using the equation:

$v_{C H}=\frac{v_{P C+C H}-\left(1-w_{C H}\right) v_{P C}}{w_{C H}}$

where, $w_{C H}$ is the mass fraction of $\mathrm{CHOL}$ in the lipidic part of the sample defined as

$w_{C H}=\frac{m_{C H}}{m_{P C}+m_{C H}}$

where, $m_{C H}$ is the mass of $\mathrm{CHOL}$ in the sample. The apparent specific volume of $\mathrm{CHOL}$ was converted to the apparent molecular volume $V_{C H}$ using the following relation:
$V_{C H}=\frac{v_{C H} M_{C H}}{N_{A}}$

where, $M_{C H}$ is the molar mass of CHOL and $N_{A}$ is the Avogadro's number.

\section{RESULTS AND DISCUSSION}

The density of samples containing multilamellar liposomes of DMPC and $\mathrm{CHOL}$ was measured in the temperature range $16-50{ }^{\circ} \mathrm{C}$. The apparent specific volume of the lipid (nonaqueous) part of samples was calculated for the control sample without $\mathrm{CHOL}, v_{P C^{\prime}}$ and for samples containing $\mathrm{CHOL}$, $v_{P C+C H^{\prime}}$ according to equations 1-4. Because the partial molecular volume of water located in bilayers is the same as the molecular volume in the bulk water (for references, see Uhríková et al., 2007), the expression 'apparent' is omitted and the fully hydrated lipid bilayers in multilamellar liposomes were treated as a separate phase. As expected, the specific volume $V_{P C+C H}$ increases with increasing temperature for various mole fractions of $\mathrm{CHOL}$ (Fig. 1).

It is well known that the hydrated bilayers of DMPC are in a solid-ordered $\mathrm{S}_{\mathrm{o}}$ phase at $t<t_{p}$ (temperature of pretransition), in a rippled $\mathrm{P}_{B}$ phase at $t_{p}<t<t_{m}$ and in a disordered fluid phase $L_{d}$ above the main phase transition temperature $t_{m}$. The main phase transition is clearly visible from the temperature dependence of $v_{P C}$ (CHOL-free DMPC bilayers). According to McMullen and et al. (1993), a small amount of CHOL is able to abolish the pretransition in DMPC bilayers. In this work, the pretransition was not included in the measured temperature

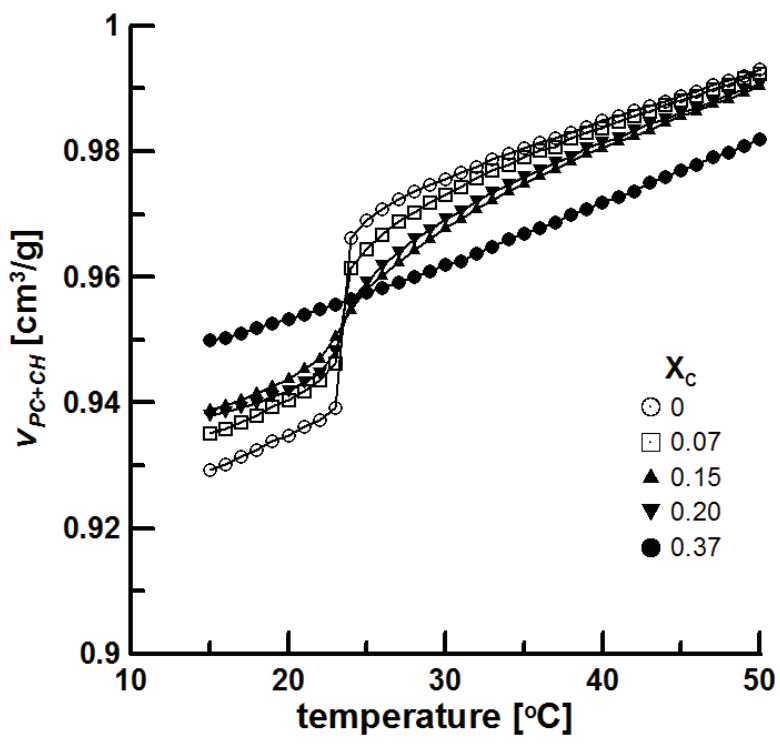

Figure 1. Temperature dependence of the specific volume of DMPC-CHOL bilayers with varying content of CHOL. Numbers denote mole fraction of $\mathrm{CHOL}$ 
range and all the samples were heated above $t_{m}$ just before the measurement to avoid a rippled $P_{B}$ phase formation. The observed main phase transition temperature $t_{m}=23-24{ }^{\circ} \mathrm{C}$ in the control DMPC sample is in agreement with data from the literature (McMullen and et al., 1993). The values of DMPC specific volume are similar to the data of Nagle \& Wilkinson (1978). The main phase transition at low CHOL mole fractions $(X<0.1)$ was narrow and only a small decrease of $t_{m}$ (within the temperature range of $1^{\circ} \mathrm{C}$ ) was observed (Fig. 1).

The transition was broadened around $X=0.1$ and was not detectable at $X \geq 0.3$ (Fig. 1). These observations are similar to those in McMullen et al. (1993). It is widely accepted that cholesterol can induce a liquid-ordered $L_{0}$ state in lipid membranes containing high amount of cholesterol. $L_{o}$ state was described by Vist \& Davis (1990) as a phase with properties intermediate between $S_{o}$ and $L_{d}$ phases. The $L_{o}$ phase is stable in a wide temperature range. We can therefore suppose that the samples with high $\mathrm{CHOL}$ content, where the main phase transition is not visible (Fig. 1), contain DMPC-CHOL bilayers in liquid-ordered $\mathrm{L}_{\mathrm{o}}$ state.

The apparent specific volume of $\mathrm{CHOL}, v_{\mathrm{CH}^{\prime}}$ was calculated (eq. $5,6)$ and converted further to the apparent molecular volume of $\mathrm{CHOL}, V_{C H}$ (eq. 7). As seen from the Fig. $2, V_{C H}$ changes when the mole fraction $X$ of $C H O L$ increases, especially at low $X$. Our preliminary results confirm the fact that $\mathrm{CHOL}$ and DMPC do not mix ideally.

In conclusion, we have determined the temperature dependence of specific volume of DMPC bilayers with various amount of $\mathrm{CHOL}$ and the temperature dependence of apparent molecular volume of $\mathrm{CHOL}$ in the mixed DMPC$\mathrm{CHOL}$ bilayers. We have confirmed a non-ideal mixing of $\mathrm{CHOL}$ and DMPC. The paper, where the partial molecular

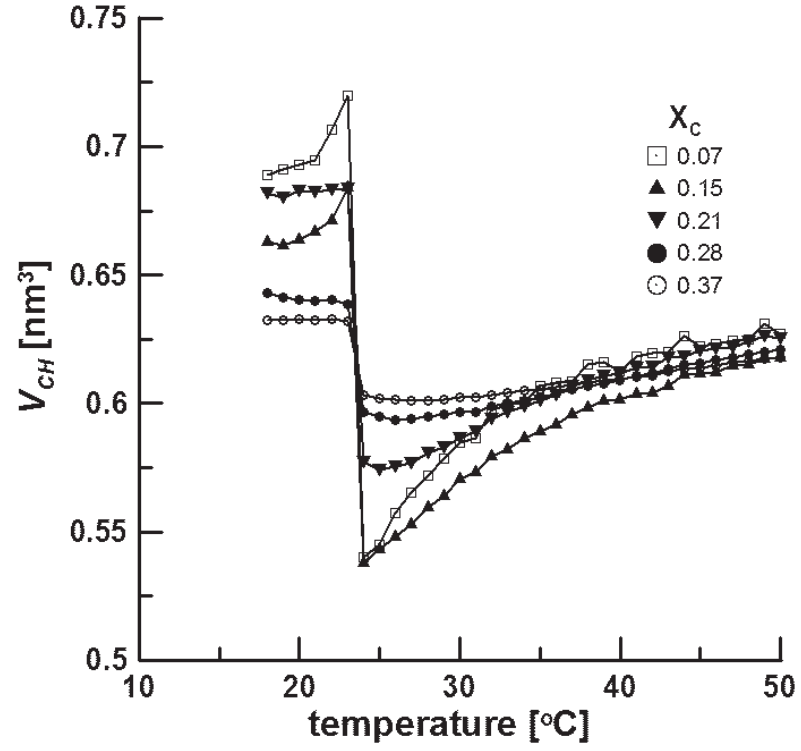

Figure 2. Temperature dependence of the apparent molecular volume of CHOL in DMPC-CHOL bilayers with varying contents of $\mathrm{CHOL}$. Numbers denote mole fraction of $\mathrm{CHOL}$

volumes of $\mathrm{CHOL}$ and DMPC will be calculated in dependence on temperature and $\mathrm{CHOL}$ content, is being in preparation.

\section{ACKNOWLEDGEMENT}

This work was supported by the Dubna JINR project 044-1121-2015/2017 and by the VEGA grant 1/0916/16 and $1 / 0228 / 17$.

\section{References}

[1] Gallová J, Klacsová M, Devínsky F, Balgavý P. Partial volumes of cholesterol and monounsaturated diacylphosphatidylcholines in mixed bilayers. Chem. Phys. Lipids 2015;190:1-8.

[2] Greenwood Al, Tristram-Nagle S, Nagle JF. Partial molecular volumes of lipids and cholesterol. Chem. Phys. Lipids 2006;143:1-10.

[3] Klacsová M, Westh P, Balgavý P. Molecular and component volumes of saturated n-alkanols in DOPC plus DOPS bilayers. Chem. Phys. Lipids 2010;163:498-505.

[4] Marquardt D, Kučerka N, Wassall SR, Harroun TA, Katsaras J. Cholesterol's location in lipid bilayers. Chem. Phys. Lipids 2016;199:17-25.

[5] McMullen TP, Lewis RN, McElhaney RN. Differential scanning calorimetric study of the effect of cholesterol on the thermotropic phase behavior of a homologous series of linear saturated phosphatidylcholines. Biochemistry 1993;32:516-522.
[6] Nagle JF, Wilkinson DA. Lecithin bilayers. Density measurement and molecular interactions. Biophys. J. 1978;23:159-175.

[7] Uhríková D, Rybár P, Hianik T, Balgavý P. Component volumes of unsaturated phosphatidylcholines in fluid bilayers: a densitometric study. Chem. Phys. Lipids 2007;145:97-105.

[8] Vist MR, Davis JH. Phase equilibria of cholesterol/dipalmitoylphosphatidylcholine mixtures: ${ }^{2} \mathrm{H}$ nuclear magnetic resonance and differential scanning calorimetry. Biochemistry 1988;29:451464. 